International Journal of Engineering \& Technology, 8(4) (2019) 527-534
International Journal of Engineering \& Technology
SPC
Website: www.sciencepubco.com/index.php/IJET
Research paper

\title{
Experimental investigation of impact damage on repetitive loading tolerance in metal-fiber multilayers
}

\author{
Banan Hasani Monfared ${ }^{1} *$, Alireza Sedaghat ${ }^{2}$ \\ ${ }^{1}$ Department of Mechanical Engineering, Lahijan Branch, Islamic Azad University, Lahijan, Iran \\ *Corresponding author E-mail: banan.monfared@gmail.com
}

\begin{abstract}
Fiber is not sensitive to fatigue in some fiber-metal multilayers. They leave a large part of the load through the cracks and prevent the crack from opening. Due to this prevention, the opening in GLARE is less than that of the metals. Unlike what is observed in metals, concentration factor of crack tip is not fully influenced by increase in crack length. Hence, this study uses the experimental method to examine post-impact fatigue behavior of glass fiber-reinforced metal composites, known as GLARE. The GLARE made in this study was produced by autoclave in three types of GLARE 1.2-3, GLARE 1.2-4 and GLARE 3.2-5 and was exposed to impact test by different forces and then fatigue test with different cycles. The results were studied. The results showed that the first GLARE 1.2-3 specimen was completely pierced after the impact test. The second GLARE 1.2-3 specimen produced fatigue cracks from impact dent in the only aluminum impacted layer. These cracks were then amplified to the edge of the specimen. Both GLARE 1.2-4 specimens showed approximately equal fatigue life. The first GLARE 1.2-4 specimen failed near the radius due to the disturbing cracks in a way that is common in FML specimen. Moreover, both GLARE 1.2-4 specimens exhibited cracking in both aluminum layers. In 1.2-5 GLARE, both specimens showed a decrease in fatigue life and increase in impact energy.
\end{abstract}

Keywords: Glass Fiber; Impact Damage; Loading Tolerance; Metal-Fiber Layers.

\section{Introduction}

Composites have been the focus of many experts in the past decades, given their many applications in various industries such as aerospace, marine, oil and gas, defense, automotive, and so on. Composites are a combination of two or more substances that are usually composed of two reinforcing phases and matrix. The reinforcing phase can be fibers, particles, or sheets that have high strength and the other phase, which is called matrix, has a preserving role and has usually high toughness [1]. Composites have become more prominent in recent decades due to many advantages they have over other alloys. These advantages include high stiffness (rigidity) relative to weight, resistance, fatigue, high erosion resistance, which lead to superior properties of composites over their components [2]. Fiberreinforced composites are also divided into two groups: integrated (continuous) and short. Strong covalent bond that is produced along the reinforcing fiber increases the modulus in this direction. The length and direction of the fibers relative to each other, their distribution as well as the percentage of fibers used in this type of composites can have a direct effect on final properties of composites. The fibers have a very high modulus in the longitudinal direction and are weak in the perpendicular direction. In order to design these types of composites in terms of size, type of stress, and level of their cost, length ratio and direction of their fibers are considered. Production speed of this type of composite is high and complex shapes can be produced which cannot be produced using continuous reinforcers.

The most important reason for failure is sudden fatigue. Failure due to fatigue occurs without any deformation and with a crisp appearance, failure surface is usually perpendicular to direction of tensile stress. The fatigue that occurs in metal-fiber laminates, such as GLARE, in its metallic part and fatigue load has no effect on its fiber part. Onset of fatigue and growth of cracks in GLARE is not due to cyclic loading, and other mechanisms of degradation, such as delamination in fiber interface, are also effective. It is assumed that the onset of crack growth can be described by linear elastic failure mechanics (LEFM) for integrated metals associated with the effect of fiber layers and delamination phenomenon [3]. Three main factors of fatigue failure include excessive maximum tensile stress, large enough or oscillating variations in stress imposed and high stress cycles. These fibers create a pathway of load transfer on the crack and prevent crack opening. As a result, less force is transferred through crack tip to metal layers, which reduces stress concentration factor of the crack tip [4]. During fatigue crack growth in metal layers, continuous distribution of stress occurs in each layer around the crack and shear stress in the interface [5]. Because of this distribution, stress concentration factor remains almost constant through a large part of the crack life. Fiber bridging mechanism depends on various factors, such as: stiffness and thickness of each layer, the number of fibermetal interfaces, direction of the adhesive fiber layer relative to loading direction, the applied load, crack configuration and environmental conditions [6]. Lamination growth is a process in which adjacent layers of cracked metal layers are laminated due to periodic shear stresses because the load is transferred to fiber-metal interface [7]. There is no tension between layers in the lamination zone, but considerable tension occurs in fibers of these layers [8]. The advantage of lamination growth is the fact that increasing the length of fiber bridging reduces the strain and stress in the fibers and prevents the fibers from exhausting. 
Fibers in some fiber-metal multilayers are not sensitive to fatigue. They cross a large part of the load through the cracks and prevent the crack from opening. Due to this obstruction, the opening in the GLARE is less than that of metals. Unlike what is observed in metals, stress concentration factor of the crack tip is not fully influenced by increase in crack length. The crack bridging reaches its maximum impact when the crack length reaches a certain value, which means that displacement reaches a certain value once the crack opens. Low crack opening means low strain on fibers and thus leads to low bridging stress. Therefore, fiber bridging and preventing crack opening for small length cracks will be low, but will be effective for cracks which reach a certain length $[7,8]$. This study experimentally examines impact damage on repeated loading tolerance in metal-fiber multilayers.

\section{Materials and methods}

\subsection{Producing the composite}

To perform this experiment, composites were produced by autoclave which is described below $[9,10]$.

1) Cutting the sheets by water jet device, stacking and dusting them

2) Washing the aluminum layers with chromic acid or phosphoric acid due to increased adhesion between aluminum layers and glass fibers

3) Cleaning the tool and aluminum layers by acetone

4) Impregnating the glass fiber layers with epoxy resin 5052

5) Organizing the aluminum layers and glass fiber based on the type of piece

6) Exposing the composites to vacuum and $0.9 \mathrm{P}$ pressure for $24 \mathrm{~h}$

7) Heating for $16 \mathrm{~h}$ at $50^{\circ} \mathrm{C}$

8) Inspecting by visual techniques and mechanical tests

\subsection{Producing and examining fiber-metal laminates}

To produce larger panels, it is required to add fittings and reinforce materials. In order to achieve a more comprehensive understanding of the process of producing and simplifying the GLAREs, fiber-metal multilayers were produced at a smaller scale in ERAU. Moreover, different types of aluminum T3-2024 with different surface properties were used to examine their differences, advantages and disadvantages properly. Glass/epoxy layers used in the experiment consisted of S-2 glass and adhesive epoxies instead of conventional FM-94 pre-impregnated materials. The main purpose of this was to lower production costs and simplify the process. Cost estimates were made by Cytec Industries (which did not include shipping and packaging costs). General costs for small values used are shown in Table 1 . Note that mat-type pre-impregnated materials are different from knit types due to random directions of their fibers. In both types, a type of strap was used for preserving fibers.

Table 1: Cost of Raw Materials and Pre-Impregnated Materials for Producing GLARE (Small Value)

\begin{tabular}{lll}
\hline Product & Value & Price \\
\hline BR-127 Primer & 1.14 liter & $172.21 \$$ \\
FM-94K (knit) & $4.65 \mathrm{~m} 2$ & $704.70 \$$ \\
FM-94-1M (mat) & $4.65 \mathrm{~m} 2$ & $545.40 \$$ \\
\hline
\end{tabular}

Eliminating the autoclave step will greatly help reduce costs. Moreover, the pressure required in the improvement step can be reduced by using lower viscosity adhesive epoxies. Quality of the surface of aluminum layers in fiber-metal multilayers will affect the quality and level of bonds when the pressure is applied; hence, surface pre-processing is a very important part in production of fiber-metal multilayers. Effects of three different methods were examined in this study: sandblasting, anodizing with phosphoric acid, and AC-130 SolGel pre-impregnated materials. Moreover, a number of shear tests were used to select the most suitable sheet for production of fibermetal multilayers.

\subsubsection{Sandblasting}

Sandblasting, also known as adhesive blasting, is a mechanical process of liquid paste abrasion on a single surface. In this process, a suitable abrasive is sprinkled on the surface by compressed air with a nozzle. In order to investigate the effects of sandblasting, small sheets of aluminum T3-2024 (25.4×50.8 mm, $0.51 \mathrm{~mm}$ in thicknesses) were subjected to shear testing. The sheets were sandblasted by a medium-sized sharp glass abrasive GA\#75 with 5.5-6 Mohs hardness (manufactured by Tacoma Company) and 60 Pa pressure in a commercial compartment. In this process, the sheets were first de-greased by multifunctional cleaner, then sandblasted, washed by acetone, and air-dried. The pre-impregnated Cytec 381 sheets were placed between the sheets. In the next step, the specimens were placed in a laboratory furnace at $80^{\circ} \mathrm{C}$ for $2 \mathrm{~h}$ (Grivo Co.); tensile stress was applied by Tinius Olsen Lo-Torq test machine.

Through the experiment, flat aluminum specimens failed before reaching the maximum load. On the other hand, the sandblasted specimens tolerated up to 2280 pounds. Although ASTM standards were not used in this experiment, sheet sandblasting improved the bonding function. The microscopic image shown in Figure 1 represents the surface of a single sandblasted sheet of aluminum T3-2024 in front of an aluminum sheet without any mechanical abrasion.
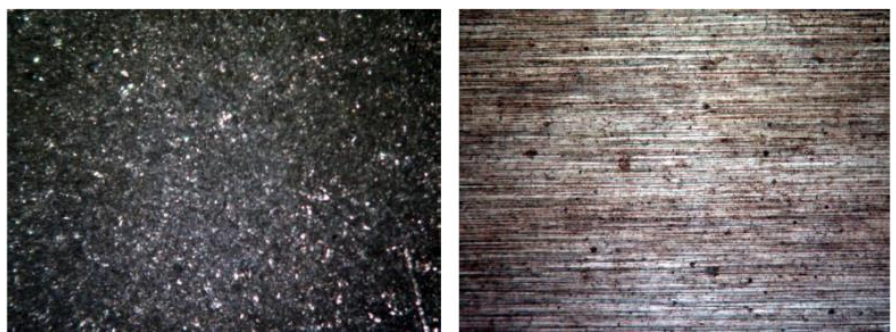

Fig. 1: Surface of the Sandblasted Aluminium T3-2024 (Left) in Comparison with A Similar Surface with Abrasion (Right) with 40x Magnification. 


\subsubsection{Anodizing by phosphoric acid}

PAA anodizing has been used in the aerospace industry since the 1970s, replacing the non-environmental chromic acid anodizing and stamping. This is done to prepare aluminum to create stronger bonds. Using this method, oxidation layers on the surface of the aluminum sheets will become thicker; these layers create mechanical bonds and locks between aluminum and adhesive. The flowchart shown in Figure 2 summarizes this process.

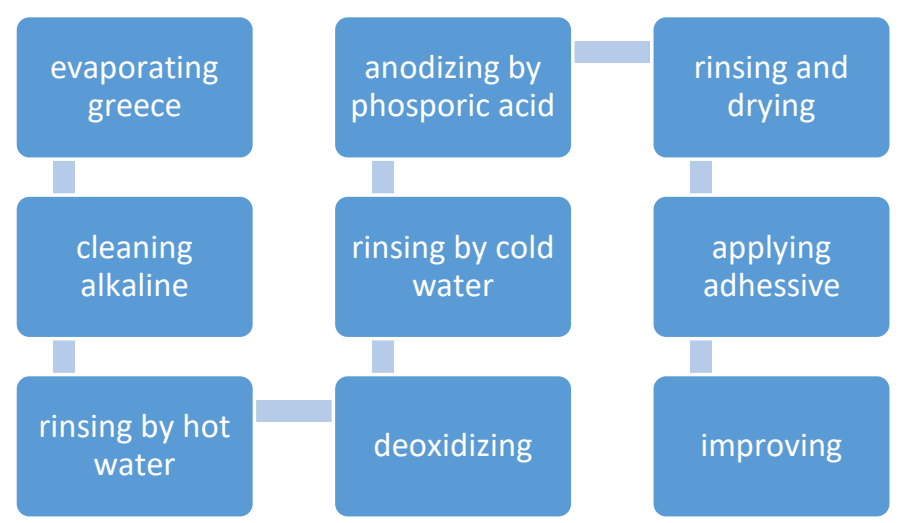

Fig. 2: PAA Process Eliminates the Need For Stamping and Using Harmful Acids.

The experiment shown in Figure 3 consists of a Cole-Parmer 8853 ultrasonic cleaner which warms the acid and allows its vibration. However, since experiments only require room temperature, this device is only used as a Pyrex container holder in which there is anode and cathode. Temperature is also monitored regularly by Omega thermocouple, Model J, connected to a meter gauge.

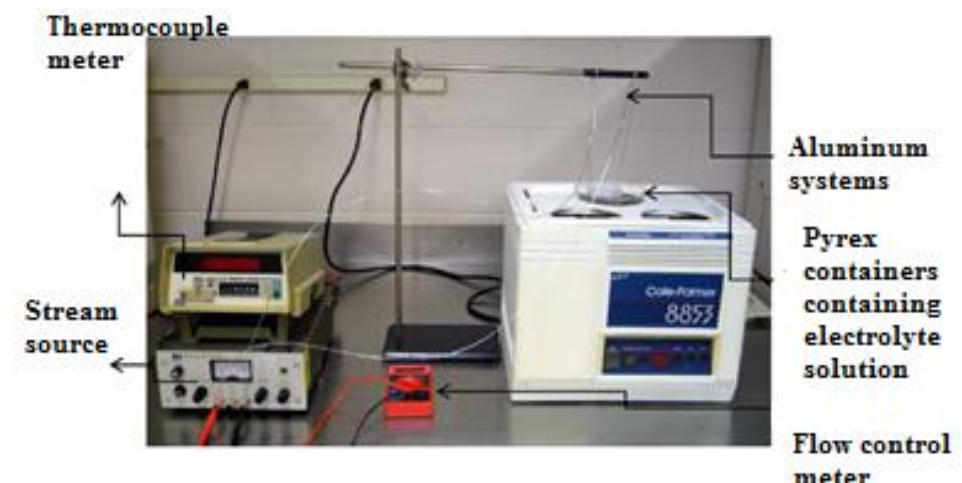

Fig. 3: PAA Setting with Voltage and Temperature Measuring Devices for Constant Monitoring.

Initially, PAA processes were performed at different times $(30 \mathrm{~min}, 1 \mathrm{~h}$ and $7 \mathrm{~h}$ ) to measure increased oxidation over time. In the first process, a $5 \mathrm{~V}$ digital voltage was used at room temperature and $\mathrm{H} 3 \mathrm{PO} 4$ with a purity of $17 \%$. $\mathrm{H} 3 \mathrm{PO} 4$ used is a product of DudaDiesel Co., with initial purity of $85 \%$. Equation (1) was used to calculate the distilled water needed to reach a purity of $17 \%$. An acid with $85 \%$ purity means $85 \mathrm{ml}$ pure acid in $100 \mathrm{ml}$ solution. Using a metallurgical microscope, the final surface was photographed, as shown in Figure 4.

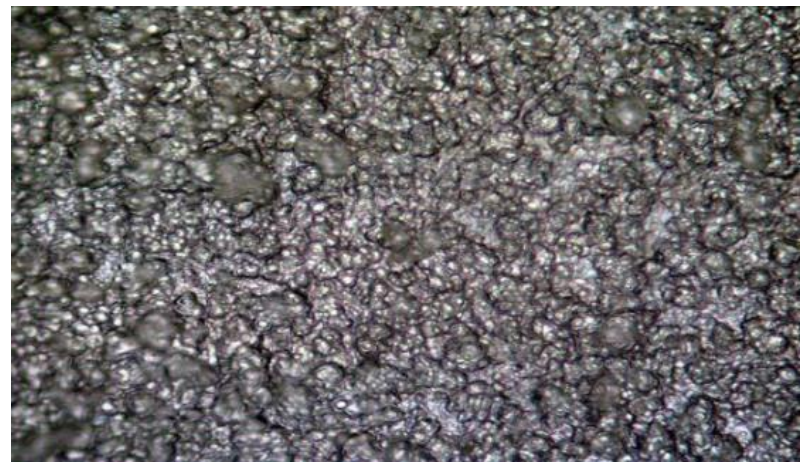

Fig. 4: Aluminum Oxide Layer Obtained From PAA with 40x Magnification

\subsubsection{AC-130 Sol-Gel}

AC-130 Sol-Gel is a product licensed by Boeing and owned by Chemistry \& Technology. Like PAA, AC-130 Sol-Gel is also used to create better bonding properties. Note that induction time refers to the time interval before activating the solution and after combining all the materials. Container lifetime also refers to the time period when solution remains usable. 


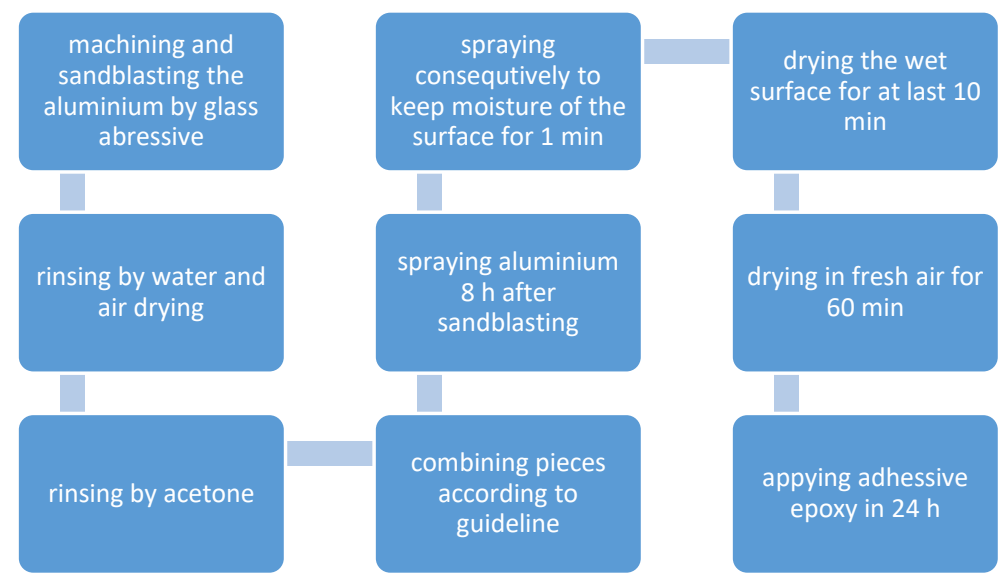

Fig. 5: Pre-Processing AC-130 Sol-Gel on Aluminum T3-2024 Sheets.

\section{Results}

\subsection{Preliminary experiments and results}

To determine adhesion bond strength and effectiveness of surface methods, shear edge tests are usually used. The goal is to find the most suitable method for preparing the aluminum surface to ensure good bonding in fiber-metal multilayers. Moreover, these experiments were repeated to compare three surface treatment methods (sandblasting, sandblasting and PAA, sandblasting and AC-130 Sol-Gel). The aluminum T3-2024 sheets (width $50 \mathrm{~mm}$ and length $200 \mathrm{~mm}, 0.5 \mathrm{~mm}$ thick) were cut and sandblasted along the edges. According to the ASTM D 5868-01 standard, the coating section of the shear edge was $25.4 \times 25.4 \mathrm{~mm}$. The ratio of resin to hardener used was also 3 to 1 . After combining materials, a brush was used to put a thin layer on the surface. Then, the specimens were stacked and placed in a vacuum bag for $6 \mathrm{~h}$ in order to provide a better bond. Details of the vacuum bag are also given in the following sections. In the next step, the specimens were removed from the bag and placed in an oven at $60^{\circ} \mathrm{C}$ for $2 \mathrm{~h}$.

Table 2: Surface Properties for Shear Edge Adhesion Testing

\begin{tabular}{lll}
\hline Details & Specimen & Method \\
\hline Equal sandblasting on the entire surface by 60 Pa pressure & 3 & Sandblasting \\
Anodizing by H2PO4 17\% for 30 min at ambient temperature & 3 & Sandblasting+PAA \\
Spraying and wetting for 5 min and drying over night & 3 & Sandblasting+Ac-130 \\
\hline
\end{tabular}

A total of 9 shear specimens were exposed to tensile stress using the Instron 8802 equipment. As before, ten values were measured for load and displacement in every second. The forehead speed was $2 \mathrm{~mm} / \mathrm{min}$. Although the recommended value by the ASTM standard for shear test was $13 \mathrm{~mm} / \mathrm{min}$, the speed in this experiment was reduced to adapt to tensile test. As shown in Figure 6, load-forehead data was converted to strain stress data.

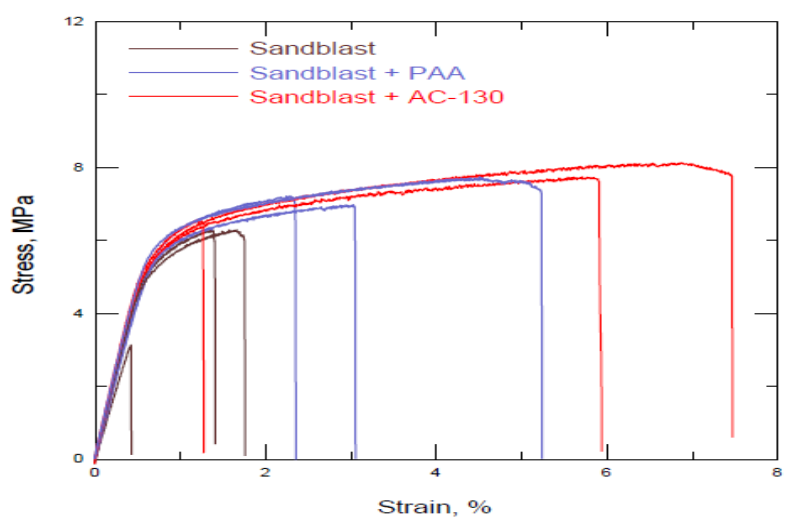

Fig. 6: Results of Shear Edge Adhesion Test between Sheets with Different Surface Properties.

For most pieces, sandblasting+AC-130 Sol-Gel method had the highest strain and shear strength. Considering the unexpected variables, such as moisture trapped in shear cover, the value obtained from stress-strain pattern could have an abnormality (a very low value for one of the AC-130 specimens). The reason for this low value is not clear. Note that metal yield might reduce shear adhesion (compared with typical shear adhesion data). For example, Table 3 shows shear strength of conventional epoxy adhesives obtained from experiments. In the next series of experiments, thicker metals were used to resolve metal failure before bond failure. By doing so, more accurate results can be obtained.

Table 3: Approximate Shear Strength of Common Epoxy Adhesive Obtained from Shear Edge Experiments

\begin{tabular}{ll}
\hline Category & Shear resistance range \\
\hline Severe & $17.2-27.6 \mathrm{MPa}$ \\
Average & $8.3-13.1 \mathrm{MPa}$ \\
Week & $<5.5 \mathrm{Mpa}$ \\
\hline
\end{tabular}




\subsection{Final experiments and results}

According to ASTM D 5868-01 standards, width of the specimens $(25.4 \mathrm{~mm})$ should remain constant during the experiment; thus, sheets of aluminum 2024-T3 and thicker (usually 5-10\% of thickness of the pure aluminum shell in both sides) that is $2.54 \mathrm{~mm}$ thick (0.10 inches) were used. Thick aluminum sheets here were chosen to prevent failure. By examining Figure 6 , it was found that sandblasting+AC-130 Sol-Gel method had better results. Hence, aluminum sheets of this experiment were also sandblasted by this method and sprayed with AC-130 Sol-Gel. Polypoxy was again used for shear overlapping. As shown in Figure 5, size of the sheets was as before $(25.4 \times 50.8 \mathrm{~mm})$. This helps to provide equal thickness of all specimens. A total of 4 shear test specimens were made. As before, shear edge test was performed on them (Figure 7).

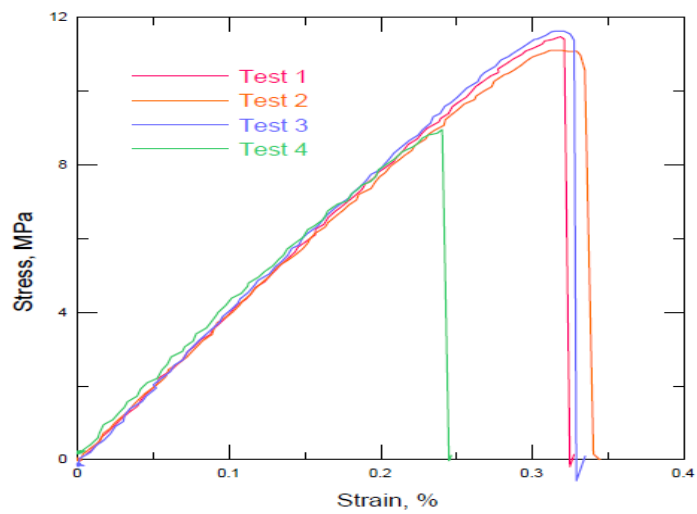

Fig. 7: Shear Edge Adhesion Test for Aluminum Alloy Alclad T3-2024 Made by Sandblasting+AC-130. Here, A 12.7 Mm Strain Change Is Due to Additional Shear Section in Experiments 3 and 4 to Measure Strain.

According to Figure 7, shear strength values were consistent with each other (with exception of experiment 4). For this anomaly, no explanation can be given. Shear forces were also very close to $12 \mathrm{MPa}$, which, according to Table 3 , would be in the higher average range. Study of the specimens following shear edge test (Figure 8) shows that adhesive was evenly distributed between the two surfaces and their attachment to each other was equal. This indicates cohesive failure (rather than adhesive failure).
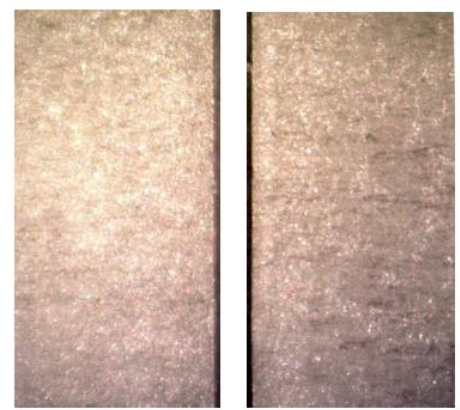

Fig. 8: Shear Edge Cohesive Failure Under A Stereozoom Microscope (Shear Zone) with A 75x Magnification.

\subsection{Epoxy adhesive}

As shown in Table 4, the information from Aircraft Spruce \& Specialty indicates that epoxies which undergo secondary improvement after the operation have better mechanical properties. It is also claimed that epoxy softening during the secondary improvement process increases molecular bonding which improves properties.

Table 4: Mechanical Properties of Polypoxy, Provided by the Manufacturer

\begin{tabular}{lll}
\hline Mechanical properties & with post-processing improvement & Without post-processing improvement \\
\hline Tensile strength & $66.2 \mathrm{Mpa}$ & $60.7 \mathrm{Mpa}$ \\
Failure strain & $7.5 \%$ & $3.6 \%$ \\
Young's modulus & $3240 \mathrm{Mpa}$ & $3172 \mathrm{Mpa}$ \\
Compressive strength & $220.6 \mathrm{Mpa}$ & $227.5 \mathrm{Mpa}$ \\
\hline
\end{tabular}

Once the hardener and the resin were mixed at room temperature, it would last for $105 \mathrm{~min}$. To determine density, a mold container was used to recover part of this epoxy (room temperature for one day and then at $60^{\circ} \mathrm{C}$ for $2 \mathrm{~h}$ ). The calculated density of this epoxy was 1.15 $\mathrm{g} / \mathrm{cm} 3$, which was obtained by using mass and volume measurements.

\subsection{S-2 glass}

Table 5 lists some properties of this glass. E, A and C versions are also fiberglass, but their resistance lies in the low to medium range. However, these fiberglasses are less costly than the S-2 glass, which makes them very suitable for cases requiring low energy.

Table 5: Mechanical Properties of S-2 Glassfibers

\begin{tabular}{ll}
\hline Property & Value \\
\hline Fiber diameter & $5-9 \mu \mathrm{m}$ \\
Power & $4585 \mathrm{Mpa}$
\end{tabular}


Two types of S-2 glass with simple and polished texture were used to produce fiber-metal multilayers instead of pre-impregnated and conventional FM-94 (lacking orientation). The goal was to make simple symmetrical sheets, without the need to use fiber, using nonorientated sheets in specific directions. This also led to lower costs. A fiber-metal multi-layered panel was made of simple texture S-2 glass and the rest of the models were made of polished texture. Important terms of woven materials include fibers, warp and fillers (woof). Fibers refer to a complex string of fiberglass threads. Warp refers to a long string of fibers along the texture and filler is also a material (or woof) that is perpendicular to the warp. Fiberglass is a simple texture used with complex warps. The shape of these two glasses is shown in Figure 9 and 10. A simple textured glass had a fiber texture; under any configuration, one passes through on top. But this happens in polished textures in more than one configuration (at least 4 ). In this particular case, the polished $\mathrm{S}-2$ glass was type 8 (eight warps), which means that the fibers pass through on one warp and under eight woofs.

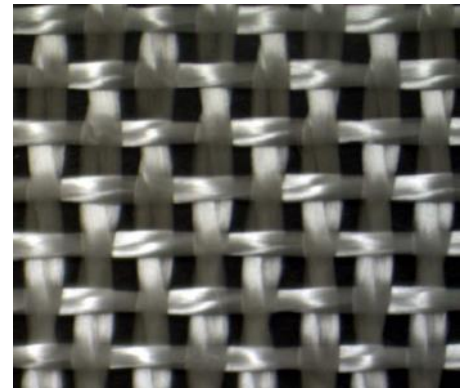

Fig. 9: Simple Texture S-2 Glass with Twisted Strings (18×18 for Each Inch) Used to Make Fiber-Metal Multilayers.

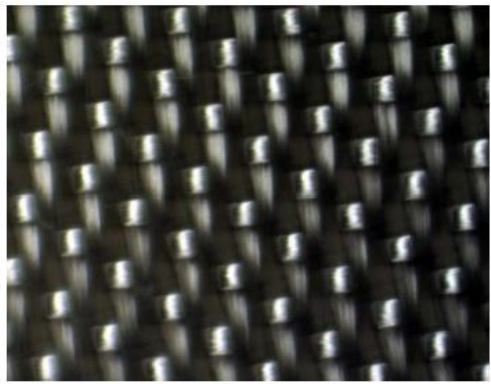

Fig. 10: Simple Texture S-2 Glass with Direct Strings $(57 \times 57$ for Each Inch) Used to Make Fiber-Metal Multilayers.

In the present experiment, two glass specimens were provided from Composites Illstreet. The simple texture 6-ounce glass cost 5.99\& per yard (and 27 inches in diameter) and the polished 8.9-ounce type cost 10.59\& per yard (and 38 inches in diameter). In Type 4533, the number of warp and woof was $18 \times 18$ per inch, while this number for the 6781 type was $54 \times 54$ or $57 \times 57$ per inch. The thickness measured for the simple textured glass was $0.23 \mathrm{~mm}$ and $0.21 \mathrm{~mm}$ for the polished type. Their density was also $0.72 \mathrm{and} 1.12 \mathrm{~g} / \mathrm{cm} 3$, respectively. Glass fabrics are usually treated during the production process to increase their adhesion properties. These treatments also lead to stronger bonding factors. The S-2 glass used had a Volan type treatment, which created a range of green color in the product. Detailed information on how the surface should be treated was not provided by the manufacturer.

\subsection{Panel construction}

Fiber-metal multilayers made used a 3/2 laminate configuration. This number means that three layers of aluminum T3-2024 and two composite layers were used to make this product. Simple aluminum sheets $(0.020$ inch or $0.51 \mathrm{~mm}$ thick $)$ were used in this product. In future studies, aluminum Alclad sheets can be an ideal option for corrosion control. In this production process, no action was taken to control corrosion in order to save costs. Each composite layer in GLARE 4B-3/2-0.4 consisted of three pre-impregnated sheets (0.127 $\mathrm{mm}$ thick), which would give a total thickness of $0.381 \mathrm{~mm}$. Since the measured thickness was $0.23 \mathrm{~mm}$ and $0.21 \mathrm{~mm}$ for simple and polished S-2 glasses, respectively, they were made of two layers of glass in production of each composite layer in fiber-metal multilayers. This configuration was chosen to increase thickness of the composite layer and make it more similar to GLASS in terms of mechanical properties. Initially, T3-2024 aluminum sheets were cut in 150×260 mm using band saws. The cut edges were also sandblasted. Then, as described in the previous section, all sheets were sandblasted and exposed to AC-130 Sol-Gel. Glass sheets, which were slightly larger than aluminum sheets, were cut to size with aluminum sheets. Their warps were so manipulated that edges of the final product became smooth.

The first aluminum sheet was placed on a flat, thick, and wax-impregnated aluminum frame covered with a Teflon sheet. In the next step, a foam brush was used to dissipate a thin layer of polypoxy on the sheet (Figure 9). Before drying the material, the S-2 glass layer was put on the aluminum sheet and its edges were adjusted. Then it was barely hit again with the brush (instead of sweeping the brush on the sheet, it was hit to avoid the sheets from moving) to wet the entire sheet. Then, extra epoxy was carefully removed of the sheets by a trowel (Figure 10). Then, another layer of fiberglass was added similarly to complete the composite layer. In the next steps, an aluminum layer, a composite layer and another aluminum layer were added to the product, respectively. This operation was performed for both S-2 glasses with simple and polished texture.

The glass/epoxy panels, which only contained two layers of S-2 glass and epoxy, were also made similarly. Then layers of fiber-metal and glass/epoxy multilayers were covered with a thin layer of Teflon sheet and a layer of respiratory sheet and placed in a vacuum bag. The vacuum bag was also tightened by a tape to the frame (the vacuum bag manufactured by Aircraft Spruce \& Specialty). To apply pressure on the panels overnight (the recommended value for this type of panel was at least $6 \mathrm{~h}$ ), a sucker $(1 \mathrm{~atm}$ or $760 \mathrm{mmHg})$ was used until the first phase of epoxy improvement was complete. In the next step, the panels were removed from the vacuum bag and placed in 
an oven at $60^{\circ} \mathrm{C}$ for $2 \mathrm{~h}$. The calculated density (using measured mass and volume) of final products can be found in Table 6 . In the next step, microscopic and tensile specimens of the same size as GLARE were made, as described.

Table 6: Calculated Density of Final Products

\begin{tabular}{ll}
\hline Specimen & Density $(\mathrm{g} / \mathrm{cm} 2)$ \\
\hline Simple textured specimen (S-2 glass and epoxy) & 1.10 \\
Polished textured specimen (S-2 glass and epoxy) & 1.61 \\
Simple texture of fiber-metal multilayers & 2.19 \\
Polished texture of fiber-metal multiplayers & 2.23 \\
\hline
\end{tabular}

\subsection{Production of specimens for the experiment}

In order to make the composite, the aluminum 2024 (0.5 mm thick), epoxy resin 5052 and $400 \mathrm{~g}$ unidirectional E-GLASS UD fiber were used. Their mechanical properties can be summarized in Tables 7,8 and 9 .

Table 7: Mechanical Properties of Aluminium 2024

\begin{tabular}{llllll}
\hline Aluminium & Modulus of elasticity (Gpa) & Ultimate stress (Mpa) & Yield stress (Mpa) & Thickness (mm) & Density \\
\hline T3-2024 & 73 & 500 & 200 & 0.5 & 2776 \\
\hline
\end{tabular}

Table 8: Properties of Resin Epoxy 5052

\begin{tabular}{lllll}
\hline Resin epoxy & KIC Mpa.m & Viscosity $25^{\circ}$ & Density g/cm3 & Lifetime (min) in $40^{\circ} \mathrm{C}$ \\
\hline LY-5052 & $0.7-0.83$ & $1000-1500$ & $1.16-1.17$ & $45-55$ \\
\hline
\end{tabular}

Table 9: Properties of E-GLASS Fibers

\begin{tabular}{lllll}
\hline Glass fibers & Fiber diameter $(\mu)$ & Fiber length $(\mu)$ & Module of elasticity $(\mathrm{Gpa})$ & Module of elasticity $(\mathrm{Gpa})$ \\
\hline E-GLASS & $17-19$ & $3-50$ & 25 & Moisture content \\
\hline
\end{tabular}

In fiber-metal composites made of 2:1 layers, upper and lower layers were made of aluminum 2024, and the middle layer was made of EGLASS glass fiber. In fiber-metal composites made of 3:2 layers, upper, lower and middle layers were made of aluminium 2024 and placed between two aluminium sheets with glass fiber. In this experiment, three types of GLARE 5-3/2, GLARE 4-2/1 and GLARE 32/1 composites were constructed; each of the four specimens were tested. Table 10 shows specifications of the constructed specimens.

Table 10: Specifications of the Constructed Specimens

\begin{tabular}{|c|c|c|c|c|}
\hline Specimen & Arrangement & Angle & Weight $(\mathrm{g})$ & Thickness (mm) \\
\hline GLARE3-2/1-11J* & AL/GL/AL & $0 / 90$ & 612 & 2.22 \\
\hline GLARE3-2/1-11J** & AL/GL/AL & $0 / 90$ & 640 & 2.27 \\
\hline GLARE3-2/1-18J* & AL/GL/AL & $0 / 90$ & 624 & 2.19 \\
\hline GLARE3-2/1-18J** & AL/GL/AL & $0 / 90$ & 633 & 2.21 \\
\hline GLARE4-2/1-11J* & AL/GL/AL & $0 / 90 / 0$ & 655 & 3.63 \\
\hline GLARE4-2/1-11J** & AL/GL/AL & $0 / 90 / 0$ & 646 & 3.35 \\
\hline GLARE4-2/1-18J* & AL/GL/AL & $0 / 90 / 0$ & 644 & 3.75 \\
\hline GLARE4-2/1-18J** & AL/GL/AL & $0 / 90 / 0$ & 652 & 3.36 \\
\hline GLARE5-3/2-11J** & AL/GL/AL/GL/AL & $0 / 90 / 90 / 0$ & 925 & 4.78 \\
\hline GLARE5-3/2-18J* & AL/GL/AL/GL/AL & 0/90/90/0 & 914 & 4.86 \\
\hline GLARE5-3/2-18J** & AL/GL/AL/GL/AL & 0/90/90/0 & 898 & 4.85 \\
\hline
\end{tabular}

\subsection{Experiment}

Initially, aluminum 2024 and composite specimens were subjected to fall impact test, the information of which is shown in Table 11. After the impact test, all specimens were exposed to fatigue load; the data is given in Table 12.

Table 11: Specifications of the Impact Test

\begin{tabular}{|c|c|c|c|c|c|}
\hline Specimen & Temperature $\left({ }^{\circ} \mathrm{C}\right)$ & Moisture (\%) & Weight (g) & Thickness (mm) & Impact energy $(\mathrm{J})$ \\
\hline Al 2024-T3-11J & 25 & 40 & 229 & 1.02 & 11 \\
\hline Al 2024-T3-18J & 25 & 40 & 218 & 1.02 & 18 \\
\hline GLARE3-2/1-11J* & 25 & 40 & 612 & 2.22 & 11 \\
\hline GLARE3-2/1-11J** & 25 & 40 & 640 & 2.27 & 11 \\
\hline GLARE3-2/1-18J* & 25 & 40 & 624 & 2.19 & 18 \\
\hline GLARE3-2/1-18J** & 25 & 40 & 633 & 2.21 & 18 \\
\hline GLARE4-2/1-11J* & 25 & 40 & 655 & 3.63 & 11 \\
\hline GLARE4-2/1-11J** & 25 & 40 & 646 & 3.35 & 11 \\
\hline GLARE4-2/1-18J** & 25 & 40 & 652 & 3.36 & 18 \\
\hline GLARE5-3/2-11J* & 25 & 40 & 892 & 4.84 & 11 \\
\hline GLARE5-3/2-11J** & 25 & 40 & 925 & 4.78 & 11 \\
\hline GLARE5-3/2-18J* & 25 & 40 & 914 & 4.86 & 18 \\
\hline
\end{tabular}

Table 12: Experimental Results of Fatigue Test

\begin{tabular}{lllllll}
\hline Specimen & $\begin{array}{l}\text { Temperature } \\
\left({ }^{\circ} \mathrm{C}\right)\end{array}$ & $\begin{array}{l}\text { Moisture } \\
(\%)\end{array}$ & $\begin{array}{l}\text { Impact } \\
\text { energy }(\mathrm{J})\end{array}$ & $\begin{array}{l}\text { Frequency } \\
(\mathrm{Hz})\end{array}$ & $\begin{array}{l}\text { Minimum loading } \\
\text { stress (Mpa) }\end{array}$ & $\begin{array}{l}\text { Maximum loading } \\
\text { stress (Mpa) }\end{array}$ \\
\hline Al 2024-T3-A & 25 & 40 & 11 & 10 & 17.5 & $\begin{array}{l}\text { Number of cycles } \\
\text { until final failure }\end{array}$ \\
Al 2024-T3-B & 25 & 40 & 18 & 10 & 17.5 & 207 \\
GLARE3-2/1- & 25 & 40 & 11 & 10 & 17.5 & 207 \\
11J* & & & & & 22822 & 207
\end{tabular}




\begin{tabular}{|c|c|c|c|c|c|c|c|}
\hline $\begin{array}{l}\text { GLARE3-2/1- } \\
\text { 11J** }\end{array}$ & 25 & 40 & 11 & 10 & 17.5 & 207 & 93855 \\
\hline $\begin{array}{l}\text { GLARE3-2/1- } \\
\text { 18J* }\end{array}$ & 25 & 40 & 18 & 10 & 17.5 & 207 & 51230 \\
\hline $\begin{array}{l}\text { GLARE3-2/1- } \\
\text { 18J** }\end{array}$ & 25 & 40 & 18 & 10 & 17.5 & 207 & 61200 \\
\hline $\begin{array}{l}\text { GLARE4-2/1- } \\
11 \mathrm{~J}^{*}\end{array}$ & 25 & 40 & 11 & 10 & 17.5 & 207 & 84921 \\
\hline $\begin{array}{l}\text { GLARE4-2/1- } \\
11 \mathrm{~J} * *\end{array}$ & 25 & 40 & 11 & 10 & 17.5 & 207 & 71750 \\
\hline $\begin{array}{l}\text { GLARE4-2/1- } \\
\text { 18J* }\end{array}$ & 25 & 40 & 18 & 10 & 17.5 & & \\
\hline $\begin{array}{l}\text { GLARE4-2/1- } \\
\text { 18J** }\end{array}$ & 25 & 40 & 18 & 10 & 17.5 & & \\
\hline $\begin{array}{l}\text { GLARE5-3/2- } \\
\text { 11J* }\end{array}$ & 25 & 40 & 11 & 10 & 17.5 & & \\
\hline $\begin{array}{l}\text { GLARE5-3/2- } \\
11 \mathrm{~J}^{* *}\end{array}$ & 25 & 40 & 11 & 10 & 17.5 & & \\
\hline $\begin{array}{l}\text { GLARE5-3/2- } \\
18 \mathrm{~J} * *\end{array}$ & 25 & 40 & 18 & 10 & 17.5 & & \\
\hline
\end{tabular}

\section{Conclusion}

Aluminum sheets failed between inspection intervals in terms of signs of cracking. On the other hand, composite sheets exhibited a sustained crack growth in the aluminum layers after onset of cracking in the impact-caused dent. Most of the FML sheets eventually failed in the curvature region of the dog's bone specimen far from diagonal cross section, because growth of the disturbing cracks in the curvature region occurred after the growing cracks reached the sheet edges from the impact-caused dent. These disturbing cracks were common in all FML sheets tested. One of the specimens was removed early from the load frame to prevent failure due to disturbing cracks. The first GLARE 1.2-3 specimen was completely pierced after the impact test. The second GLARE 1.2-3 specimen caused fatigue cracks from impact dent in the only aluminum impacted layer. These cracks were then amplified to the specimen edge. As a result, disturbing cracks were created. This sample failed due to disturbing cracks in the radius. Both GLARE 1.2-4 specimens showed an approximately equal fatigue life. The second specimen actually failed due to cracking. This crack was in direction of the sheet loading and its center was in the dent. This damage behavior is due to impact in FML with different fiber volume portions in two vertical directions. This crack did not grow during the fatigue test. However, the second crack began in the dent and propagated parallel to the loading direction. When the crack reached the edge of the dent region, its growth stopped. This specimen was removed before the disturbing cracks caused complete failure. The first GLARE 1.2-4 specimen failed near the radius in a typical FML specimen due to the disturbing cracks. Both GLARE 1.2-4 specimens showed cracking in both aluminium layers. In GLARE 1.2-5, both specimens showed reduced fatigue life and increased impact energy. The first specimen was removed before complete failure. The second GLARE 1.2-5 specimen failed in radius due to disturbing cracks.

\section{References}

[1] Buck A, "Fatigue properties of pure metals", International Journal of Fracture, Vol. 3, (1967), pp. 145-152. https://doi.org/10.1007/BF00182692.

[2] Botelho EC, Silva RA, Pardini LC, \& Rezende MC, "A review on the development and properties of continuous fiber/epoxy/aluminum hybrid composites for aircraft structures", Materials Research, Vol. 9, No. 3, (2006), pp. 247-256. https://doi.org/10.1590/S1516-14392006000300002.

[3] Laird C, and Krause AR, "A theory of crack nucleation in high strain fatigue", International Journal of Fracture, Vol. 4, (1968), pp. $219-231$. https://doi.org/10.1007/BF00185258.

[4] Asundi A, \& Choi AY, "Fiber metal laminates: an advanced material for future aircraft", Journal of Materials processing technology, Vol. 63, No. 1-3, (1997), pp. 384-394 https://doi.org/10.1016/S0924-0136(96)02652-0.

[5] Kawai M, \& Hachinohe A, "Two-stress level fatigue of unidirectional fiber-metal hybrid composite: GLARE 2", International Journal of Fatigue, Vol. 24, No. 5, (2002), pp. 567-580. https://doi.org/10.1016/S0142-1123(01)00108-6.

[6] Alderliesten RC, Fatigue crack propagation and delamination growth in Glare, (2005).

[7] Dadej K, Bieniaś J, Surowska B, "Residual Fatigue Life of Carbon Fiber Aluminium Laminates", International journal of Fatigue, (2018) https://doi.org/10.1016/j.ijfatigue.2017.03.026.

[8] Dadej K, Surowska B, \& Bieniaś J, "Isostrain elastoplastic model for prediction of static strength and fatigue life of fiber metal laminates", International Journal of Fatigue, Vol. 110, (2018), pp. 31-41. https://doi.org/10.1016/j.ijfatigue.2018.01.009.

[9] Amini M, Khavandi A, "Degradation of polymer-based composites in corrosive media: experimental attempts towards underlying mechanisms", Mechanics of Time-Dependent Materials, Vol. 23, 2(2019), pp.153-172. https://doi.org/10.1007/s11043-018-09408-7.

[10] Amini M, Khavandi A, "Synergistic effects of mechanical and environmental loading in stress corrosion cracking of glass/polymer composites", Journal of Composite Materials, Vol. 53, 24 (2019), pp. 3433-3444. https://doi.org/10.1177/0021998319842376. 